\title{
Mögliche Effekte der Digitalisierung für die Inflationsrate
}

Seit dem Beginn der 1990er Jahre haben sich die Preise für die meisten Produkte aus dem Bereich der Informations- und Kommunikationstechnologie erheblich verringert. Für die Konsumenten bedeutet dies niedrigere Verbraucherpreise und damit auch eine geringere Inflationsrate. ${ }^{1}$ Der Einsatz von Computern, Robotern, künstlicher Intelligenz und anderen digitalen Technologien bei der Herstellung von Gütern und Dienstleistungen hat darüber hinaus die Produktivität gesteigert, z. B. durch die Beschleunigung betrieblicher Prozesse (z. B. Produktentwicklung, Verwaltung, Handel und Vertrieb sowie das gesamte Beschaffungswesen) aufgrund von Automatisierung und Algorithmisierung, durch zielgerichtetere Kundeninformation aufgrund von personalisierter Werbung und durch eine Verringerung von Fehlern in der Produktion aufgrund von automatisierten Produktionsverfahren. Die Optimierung dieser Prozesse reduziert Verwaltungs-, Entscheidungs- und Produktionskosten, was im Normalfall eine Preissenkung nach sich zieht.

Inflationsdämpfend wirkt auch die Ausbreitung der Sharing Economy und der eng damit verbundenen digitalen Plattformen, vor allem aus zwei Gründen:

- Zum einen steigt das Angebot im informellen Bereich. Beispiele für solche Plattformen sind Airbnb und Uber. Der daraus resultierende Angebotsüberschuss führt zu

(C) Der/die Autor(en) 2020. Open Access: Dieser Artikel wird unter der Creative Commons Namensnennung 4.0 International Lizenz (http:// creativecommons.org/licenses/by/4.0/deed.de) veröffentlicht.

Open Access wird durch die ZBW - Leibniz-Informationszentrum Wirtschaft gefördert.

1 Vgl. K. Charbonneau et al.: Digitalization and Inflation: A Review of the Literature, Bank of Canada: Staff Analytical Note 2017-20, Ottawa 2017, S. 3.

Dr. Thieß Petersen ist Senior Advisor der Bertelsmann Stiftung in Gütersloh im Projekt „Global Economic Dynamics“ und Lehrbeauftragter an der EuropaUniversität Viadrina in Frankfurt an der Oder. einem Preisrückgang, der sich auf die kommerziellen Anbieter auswirkt. Verstärkt wird dieser Preisdruck dadurch, dass private Anbieter geringere Standards berücksichtigen müssen als kommerzielle Anbieter und deshalb auch niedrigere Preise fordern.

- Zum anderen kann die Sharing Economy einen Nachfragerückgang bewirken. Wenn sich im Rahmen des Carsharings mehrere Personen ein Fahrzeug teilen, sinkt die Zahl der nachgefragten Autos. Auf dem Automobilmarkt ruft das einen Angebotsüberschuss mit einem entsprechenden Preisrückgang hervor.

Auch der digitalisierungsbedingte Abbau von Marktmacht lokaler Anbieter hat einen preisniveausenkenden Effekt. Durch den Preisvergleich im Internet können Kaufinteressierte unmittelbar sehen, ob sie bei inrem Anbieter vor Ort einen höheren Preis als Online zahlen. Lokale Anbieter müssen sich folglich an die niedrigeren überregionalen Preise anpassen. Dieser höhere Wettbewerbsdruck sorgt dafür, dass Unternehmen Preisaufschläge, die ihren Gewinn steigern, abbauen müssen. ${ }^{2}$ Die faktische Relevanz dieses Effekts für die Inflationsrate ist momentan noch gering. Nach einer Untersuchung der Europäischen Zentralbank hat "die zunehmende Nutzung des OnlineHandels in der EU die Inflationsrate bei Industriegütern (ohne Energie) seit 2003 jährlich um 0,1 Prozentpunkte gesenkt" ${ }^{\text {"3 }}$. Bei einer Ausdehnung des Online-Handels ist es durchaus vorstellbar, dass diese preissenkende Wirkung perspektivisch größer wird. Einschränkend ist jedoch zu berücksichtigen, dass dieser Effekt einmalig ist: Er baut die Preisaufschläge auf die Produktionskosten ab. Bei vollständiger Markttransparenz mit vielen Anbietern verschwinden diese Aufschläge. Ist dieser Zustand erreicht, „wären die Preissenkungspotenziale ausgeschöpft und der Einfluss auf die Inflationsrate verschwunden“4. 
Preisniveausenkend wirkt zudem der mit der Digitalisierung verbundene Trend zur Prosumtion, also die stärkere Einbindung des Verbrauchers in die Produktionsprozesse. ${ }^{5}$ Wenn Konsumenten im Internet Elektrogeräte kaufen oder Reisen buchen, übernehmen sie die Beratungsdienstleistungen in Elektrogeschäften und Reisebüros. Unternehmen erbringen diese Arbeitsschritte nicht mehr selbst. Damit sinken ihre Produktionskosten und mit ihnen die Marktpreise.

Schließlich sind die Arbeitsmarkt- und Lohneffekte der Digitalisierung zu beachten. Zumindest mittel- und langfristig ist es in entwickelten Industrieländern durchaus plausibel, dass die Freisetzungseffekte der Digitalisierung höher sind als deren Kompensationseffekte, was zu einem gesamtwirtschaftlichen Beschäftigungsrückgang führt. ${ }^{6}$ Das bewirkt einen tendenziellen Lohndruck, der die Inflationsrate dämpft. Grundsätzlich können digitalisierungsbedingte Preisrückgänge auch die Lohn-Preis-Spirale abbremsen. Die genannten Entwicklungen (technologischer Fortschritt, Angebotsausweitung und Abbau von Marktmacht) verhindern einen Preisanstieg. Zum einen sind nur geringe Nominallohnerhöhungen erforderlich, um einen inflationsbedingten Kaufkraftverlust zu kompensieren. Zum anderen drückt die nachlassende Nachfrage nach Arbeitskräften auf den Lohn. Beide Effekte dämpfen die Inflation.

\section{Preisniveauerhöhende Effekte der Digitalisierung}

Neben den inflationssenkenden Effekten kann die Digitalisierung allerdings auch preiserhöhende Auswirkungen haben (vgl. Tabelle 1). Drei Effekte sind hier von besonderer Bedeutung.

1. Die voranschreitende Digitalisierung hat die Tendenz, natürliche Monopole hervorzubringen. Eine Ursache dafür ist die spezielle Kostenstruktur vieler digitaler Güter und digitaler Plattformen: Während die Entwicklung dieser Güter bzw. der Aufbau der Plattformen mit hohen Fixkosten verbunden ist, tendieren die Grenzkosten der Produktion gegen null. Zudem haben viele digitale Produkte den Charakter eines Netzwerkguts. Dies bedeutet: Je mehr Teilnehmer in einem sozialen Netzwerk oder einer Online-Tauschbörse anzutreffen sind, desto attraktiver ist es, sich dem entsprechend großen Netzwerk anzuschließen. Langfristig setzt sich somit der Anbieter durch, der die meisten Teilnehmer hat. In der Digitalökonomie besteht somit „die Tendenz, dass natürliche Monopole zum Normallfall

5 Vgl. K.-U. Hellmann: Prosumer Revisited: Zur Aktualität einer Debatte - Eine Einführung, in: B. Blättel-Mink, K.-U. Hellmann (Hrsg.): Prosumer Revisited: Zur Aktualität einer Debatte, Wiesbaden 2010, S. 14-16.

6 Vgl. T. Petersen: Diginomics verstehen - Ökonomie im Licht der Digitalisierung, München 2020 (im Erscheinen), Kap. 7.
Tabelle 1

Verschiedene Effekte für die Inflationsmessung

Preisniveaureduzierende Effekte der Digitalisierung

Sinkende Preise von Informations- und Kommunikationstechnologieprodukten

Digitalisierungsbedingte Produktivitätssteigerungen

Ausweitung der Sharing Economy und digitaler Plattformen

Abbau von Marktmacht lokaler Anbieter (Amazon-Effekt)

Trend zur verstärkten Prosumtion

Freisetzungseffekte der Digitalisierung auf dem Arbeitsmarkt

Kostenlose Bereitstellung von Produkten

Digitalisierungsbedingte Qualitätsverbesserungen von Produkten

Preisniveauerhöhende Effekte der Digitalisierung

Digitale natürliche Monopole

Kartellbildung durch algorithmische Preisbildung

Personalisierte Preise

Kompensationseffekte der Digitalisierung auf dem Arbeitsmarkt

Quelle: eigene Darstellung.

werden“7. Die Folge ist ein Monopolpreis, der über dem Preis bei vollständiger Konkurrenz liegt und somit das Preisniveau erhöht.

2. Der verstärkte Einsatz von Algorithmen kann zur Bildung von Preiskartellen führen, in dem sich die Mitglieder auf einen Preis, der über dem der vollständigen Konkurrenz liegt, einigen. Damit es nicht zu einem Angebotsüberschuss kommt, der einen Preisrückgang hervorrufen würde, ist ein Kartell gleichzeitig mit einer Festlegung der Menge verbunden, die jeder Einzelanbieter zu dem vereinbarten Preis auf den Markt bringen darf. Wenn viele Unternehmen nun Algorithmen in ihrem Preisfindungsprozess einsetzen, kann diese Software zu einem kartellanalogen Preis führen, der inflationssteigernd wirkt. ${ }^{8}$

3. Schließlich können auch personalisierte, also kundenindividuelle Preise, die Online-Anbieter von unterschiedlichen Personen für identische Produkte verlangen, preiserhöhend wirken. Die Höhe des personalisierten Preises hängt von nutzerbezogenen Eigenschaften $a b$, z.B. sozioökonomische Eigenschaften wie Alter und Geschlecht, bisheriges Such- bzw. Surfverhalten und verwendetes Endgerät. ${ }^{9}$ Unternehmen erhöhen ihren Gewinn, indem sie von unterschiedlichen Käufern

7 J. Quitzau, A. Broders: Marktwirtschaft in Gefahr? Digitale Monopole, Hamburg 2019, H. S. 1.

8 Vgl. J. Haucap: Daten als Wettbewerbsfaktor, in: Wirtschaftsdienst, 98. Jg., (2018), H. 7, S. 475.

9 Vgl. Verbraucherzentrale Bundesverband: Personalisierte Preise, Diskussionspapier des Verbraucherzentrale Bundesverbands, Berlin 2016, S. 3. 
verschiedene Preise fordern. Dabei versuchen sie, die maximale Zahlungsbereitschaft der Kunden so weit wie möglich auszunutzen. Für die Volkswirtschaft bedeutet das letztendlich ein höheres Preisniveau. Auch wenn das Phänomen der personalisierten Preise bisher nur vereinzelt nachweisbar ist, ${ }^{10}$ könnte es mit der Weiterentwicklung der Datensammlung und der Datenauswertung an Bedeutung gewinnen.

\section{Messprobleme der offiziellen Statistiken}

Ein zentraler Grund für den inflationsdämpfenden Effekt der Digitalisierung besteht aus den von digitalen Technologien verursachten Produktivitätsfortschritten. Der Wirtschaftsnobelpreisträger Robert Solow wies bereits 1987 darauf hin, dass wir die produktivitätssteigernden Wirkungen von Computern überall sehen - nur nicht in den offiziellen Produktivitätsstatistiken. ${ }^{11}$ Ein Grund hierfür sind Messfehler der Volkswirtschaftlichen Gesamtrechnung (VGR) bei der Erfassung der wirtschaftlichen Wertschöpfung. Hier ist vor allem auf zwei Fehler hinzuweisen:

Tendenz zur kostenlosen Bereitstellung von Produkten: Die Digitalisierung ersetzt Produkte, für die die Verbraucher bisher einen Preis zahlen mussten (Zeitungen, Bücher, CDs, Beratungen im Reisebüro etc.), durch kostenlose digitale Angebote (Online-Portale, Wikipedia, kostenlose Apps etc.). Da die VGR nur Güter und Dienstleistungen berücksichtigt, für die ein Marktpreis gezahlt wird, werden die kostenlosen digitalen Produkte nicht erfasst. So werden der mengenmäßige Output und die Produktivitätsfortschritte unterschätzt.

Unzureichend erfasste Qualitätsverbesserungen: Der technische Fortschritt verbessert häufig die Qualität von Produkten, was ebenfalls wie eine Preissenkung wirkt: Bleibt der Preis eines Produkts bei verbesserter Qualität konstant, erhält der Verbraucher für den gleichen Preis eine bessere Qualität. Umgekehrt bedeutet dies, dass der Verbraucher für eine unveränderte Qualität einen geringeren Preis zahlen muss. Werden diese Qualitätsverbesserungen bei der Berechnung der Produktivität nicht vollständig erfasst, werden Produktivitätszuwächse unterschätzt. Dies ist besonders gravierend, wenn digitale Technologien andere Produkte vom Markt verdrängen: Ein Handy oder Smartphone ist zunächst einmal ein Kommunikationsgerät zusätzlich zum Festnetzanschluss. Zusatzfunktionen können jedoch andere Produkte verdrängen - wie z.B. Fotoapparate und Videokameras, Filme und deren Entwicklung sowie Fo-

10 Vgl. H. Zander-Hayat, I. Domurath, C. Groß: Personalisierte Preise, SVRV Working Paper (2), Berlin 2016, S. 2.

11 Vgl. R. Solow: We'd better watch out, in: New York Times vom 12.7.1987, S. 36 . toalben. Um die Produktivitätsentwicklung im Zeitablauf vergleichen zu können, müssten alle diese Produkte in die Messung des realen Bruttoinlandsprodukts (BIP) einbezogen werden. Geschieht dies nicht vollständig, weist das BIP eine zu geringe wirtschaftliche Wertschöpfung aus.

Somit gibt es also „einen robusten positiven Zusammenhang zwischen Digitalisierung und Produktivität", der durch zahlreiche Studien belegt ist. ${ }^{12}$ Nicht alle Produktivitätssteigerungen und Kostenreduzierungen werden jedoch vollständig in den offiziellen Statistiken abgebildet und damit unterschätzt. Die tatsächliche Inflationsrate ist geringer als die offiziell ausgewiesene. Dieser Zusammenhang gilt als Konsens. Wie stark diese Messfehler ins Gewicht fallen, ist unter Ökonomen jedoch umstritten. ${ }^{13}$ Entsprechende Messfehler gelten auch für die inflationserhöhenden Effekte der Digitalisierung. So spielen die von einem natürlichen digitalen Monopol zu erwartenden Preiserhöhungen gegenwärtig keine spürbare Rolle, weil die meisten Dienstleistungen von digitalen Plattformen kostenlos bereitgestellt werden (zumindest mit Blick auf eine monetäre Gegenleistung). Es ist jedoch durchaus denkbar, dass die Kunden mit der Preisgabe ihrer Daten einen überhöhten Monopolpreis zahlen. ${ }^{14}$

Auch wenn die Digitalisierung teilweise inflationserhöhend wirkt, dürften die preisniveausenkenden Effekte per Saldo überwiegen. Vor allem der mit den digitalen Technologien einhergehende Rückgang der Produktionskosten sollte die Inflationsraten niedrig halten. Bisher ist der inflationsdämpfende Einfluss der Digitalisierung jedoch relativ gering, weil sie in weiten Bereichen immer noch in einer frühen Entwicklungsphase steckt und folglich nicht alle preisniveausenkenden Konsequenzen zum Tragen kommen. Hinzu kommt, dass die Statistiken der VGR zwar mit methodischen Verbesserungen auf die Digitalisierung reagieren, ${ }^{15}$ aber dennoch Schwierigkeiten haben, Preise (und Mengen) der digitalen Güter richtig zu erfassen. Es gibt daher auch die These, dass in entwickelten Volkswirtschaften bereits eine digitale Deflation herrschen könnte, die jedoch von den offiziellen Statistiken nicht erfasst wird. ${ }^{16}$

12 Vgl. K. Lichtblau, M. Fritsch, A. Millack: Digitalisierung von Wirtschaft und Gesellschaft in Deutschland - Ein Überblick, Köln 2018, S. 77

13 Vgl. A. Brandt: Digitaler Kapitalismus - Dynamiken, Potenziale, Monopole, in: spw, Heft 220, 3/2017, S. 39-47, hier: S. 43.

14 Vgl. J. Krämer: Datenschutz 2.0 - ökonomische Auswirkungen von Datenportabilität im Zeitalter des Datenkapitalismus, in: Wirtschaftsdienst, 98. Jg., 2018, S. 466-469, hier: S. 466.

15 Z.B. mit einer hedonischen Qualitätsbereinigung und dem sogenannten Web-Scraping. Letzteres ist eine computergestützte automatisierte Preiserhebung im Bereich des Online-Handels. Vgl. D. Schäfer, M. Bieg: Auswirkungen der Digitalisierung auf die Preisstatistik, Methodeninformation des Statistischen Bundesamts, Wiesbaden 2016.

$16 \mathrm{Vgl}$. G. Hutchins: What if we are all wrong on productivity?, in: Financial Times vom 29.1.2020, S. 9. 